\title{
Futures for computer-based training: Developing the learner-computer interface
}

\author{
Rod Sims \\ Department of Information Studies \\ Kuringai CAE
}

\begin{abstract}
Often regarded as the solution to effective skills development, ComputerBased Training (CBT) is now satisfying many training demands. However, despite continued developments with instructional technology in areas such as embedded CBT and intelligent instructional systems, CBT remains a support mechanism for staff development rather than an integral component of computer-based information systems, and often fails to address the individual attributes of the learner. A model is proposed integrating the advanced components of intelligent CBT (interaction, individualisation, availability) based on human factors issues to provide a user-based operational environment. A second section appraises six variables (instructional design, technology, cognitive style, screen design, transfer and retention, and learner-computer interface) to describe an updated methodology for the design, development and implementation of technologically-advanced CBT systems. By reinforcing the capabilities of CBT and integrating these with changes in information technology, the scene is set for development and growth in learning through computerbased and learner-centred educational systems, founded on contextsensitive learner-computer interface.
\end{abstract}

Almost daily, the capability of computer technology to perform more quickly, access more information and outclass existing software applications is reported. However, users of the technology have changed very little, except perhaps in expressing increased frustration at the inability of applications to meet individual skill levels (Martin, 1986b).

The potential of Computer-Based Training (CBT) to address this environment is the topic of this paper, through an evaluation of two 
options for CBT implementation which address the individual user and learner. The first is based on the converging paths of instructional technology, systems design methodologies and human factors, considered with reference to developments in educational technology based on Embedded CBT (Bentley, 1988) and Intelligent Computer-Assisted Learning (Kearsley, 1987), to describe a model which integrates these elements into an adaptive computer-based system catering for the varying expertise and learning styles of individual users. The second examines selected factors in the courseware development process which are crucial to the implementation of resources which maximise the transparency of the learner-computer interface.

\section{The Learner-Computer Interface I: Towards integrated adaptive application software}

In the data processing environment, the demand for a user-interface flexible enough to meet novice and expert needs, consistent in its presentation and adaptable to individual skill levels is of increasing importance (Davis and Olson, 1985; Martin, 1986a,b). Developments in integrating human factors to software applications include adaptive models which cater for individual skill levels (Martin 1986b) and cognitive psychology issues relating to the ways people process information (Gardiner and Christie, 1987). This interest in adaptive systems and cognitive science is not only applicable to human factors, but also to CBT applications.

With the proliferation of technology, user knowledge and skills are the concern of training, and therefore provide an opportunity to integrate training into the development of software systems. Significantly, these assumptions extend across the range of software packages and highlight the opportunity to develop skills in user-interface or communication.

Interestingly, the technology itself is rarely used to support this training, other than providing the means to operate software in classroom settings (Sims, 1986). More recently, CBT has been implemented in an attempt to facilitate the availability of training, with considerable success. In fact, the support of application software with CBT would appear to be an attractive alternative for both vendor and user (Sims, 1988b).

\section{Computer-Based Training (CBT)}

Computer-Based Training (CBT) has developed to be recognised as an effective solution for skills training. However, despite the accepted instructional benefits such as individual learning and self-pacing, CBT is only now beginning to make a significant impact on training practices (Sims and Grant, 1987). The apparent reason for this delay is the development costs associated with instructional software and the lack of 
identified training needs to justify the development effort. In fact, instructional technology often appears to be used for its novelty rather than instructional worth, emphasised by the suggestion that only tomorrow's technology will provide effective and efficient CBT (Kearsley, 1987).

Nevertheless, in the Australian business environment, there is a growing demand for trained employees, based on the assumption that skilled operation will result in a minimisation of errors and increased productivity. A basic form of CBT which has been adopted in software applications to assist the novice user are the on-line or "hot-key" HELP facilities (Sims, 1988a). However, these only provide information to the user rather than training in skill deficiency; additionally, the information displayed often assumes a certain level of user knowledge to allow interpretation of the HELP message.

A future development for this concept will be the HELP "window" which describes more accurately the item requested, as well as providing the opportunity for further Computer-Based Training. The only drawback to this approach will be the development effort required for implementation (in addition to those for human factors as described by Mantei and Teorey, 1988) and possible hardware and software constraints, such as memory, storage or response times. There are of course many areas where CBT has been used to assist with developing skills in using application software (Sims, 1988b). However, CBT has typically been used in parallel with the software application for which it has been applied. One of the more recent developments, and incorporating the "extended-HELP" identified above, is the concept of Embedded Computer-Based Training (ECBT) which does not distinguish between the software application and the training system, but rather integrates the two in the system development cycle.

\section{Embedded Computer-Based Training (ECBT)}

Embedded CBT (ECBT) has the benefit of being delivered to the user/trainee on a "live" rather than an off-line training system.

ECBT is quite definitely best employed to train people to make effective use of the technology they use in their daily work. It does this by utilising the same technology used in the workplace as the main training tool (Bentley, 1988, p19)

The major elements of ECBT include a training system, an operational system, an interactive help system, a training database and an activity monitoring facility (Bentley, 1987). With these components the user has the option to access training which is supervised by the software application to control the communication between the user/trainee, the training database and the training system. 
The interactive help system is always available for on-screen assistance to the user. The training database is maintained to reflect the actual operational system and the monitoring facility provides feedback information relating to the effectiveness and use of the training system. The latter component is essential for effective training as it provides data for the modification of the training to meet changes in learner needs or system characteristics.

ECBT requires a novel approach in that trainers, systems analysts and users must all form part of the design team; these individuals define the project group whose task is to analyse the software applications well as the training requirements. The significant advance in training technology is that training based on ECBT is designed at the same time as the application software, and the training itself forms part of the operational system. The importance of this approach is that rather than training users about the technology, ECBT involves learner-centred training using an integrated training system. In essence, ECBT modifies the application of human factors to systems design (Mantei and Teorey, 1988) to include training and learner factors.

While ECBT provides immediate access to training based on the active system, there is no guarantee that it will cater for the individual skill levels of users or their particular learning requirements. This leads to a consideration of advancements in software technology which are bringing training systems, information systems and human factors closer and closer together by the integration of artificial intelligence and expert systems to produce intelligent computer-assisted learning resources (Black, 1986; Kearsley, 1987).

\section{Intelligent Computer-Assisted Learning (ICAL)}

The development of Intelligent Computer-Assisted Learning (ICAL) provided a new era for computer-based learning systems, with the potential for instruction based on an individual's particular needs. The significant difference between ICAL and traditional CBT is the move from teacher-based systems towards learner-based systems, such that the instructional resource can draw on a knowledge-base (content) by a tutorial model or inference engine which is activated by trainee responses. The content presented to students is dependent upon their particular circumstances rather than those defined by an instructor.

One of the significant research areas in artificial intelligence is the natural language interface whereby users can communicate with the system using everyday language. The benefits of this in terms of human factors and software applications is the option for users to make inquiries about a particular operation using their own terms and whatever their particular skill. Currently however, the availability of such systems is restricted by 
the capability of the technology to communicate with the user on an "intelligent" basis.

The desirability of natural language as a communication medium must be considered in terms of software engineering, which is currently far from providing this facility. However, in discussing options for "intelligent" training, the possibilities can be extended to those in Figure 1.

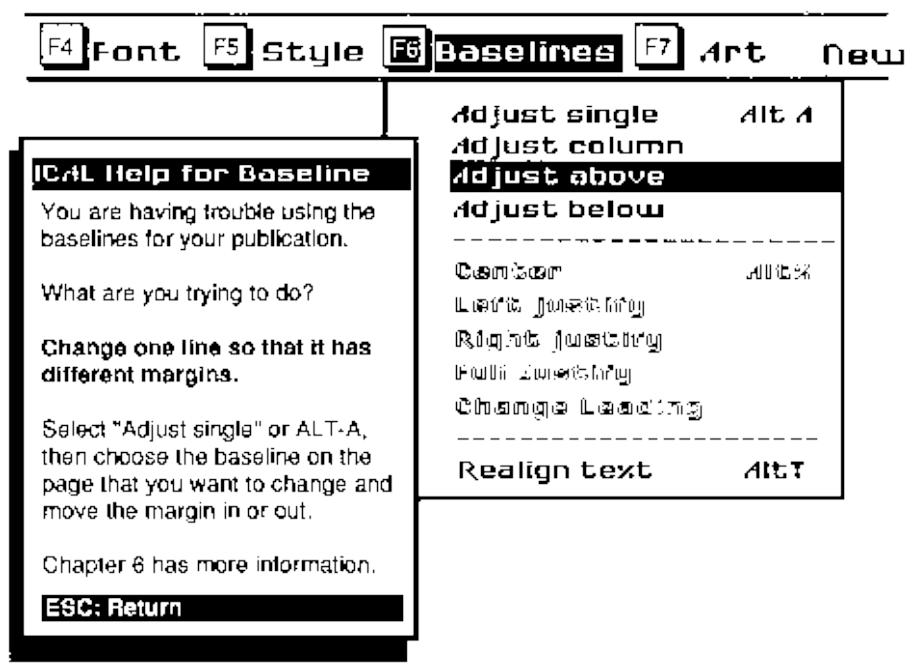

Figure 1: Intelligent Computer-Assisted Learning

In this example, Software Publishing's First Publisher would be modified to include a record of tasks which cause problems for individual users. Once a user attempts a pre-defined number of incorrect or unavailable options, the system will respond with guidance and a request for clarification. The user may then pose a question (shown in bold type) which the application, through natural language processing, will interpret and make a response. While this example is purely hypothetical, it illustrates the direction of user training and the convergence of human factors and systems development issues.

Like traditional and embedded CBT applications, Intelligent Computer Assisted Learning (ICAL) has often been used in isolation from the system for which it is providing the training. However, the benefit of ICAL is its ability to adjust and modify training to cater for individual levels of knowledge and understanding. In doing so, it draws upon a particular knowledge base, a model of the current student and a tutorial model which can determine the next step in the instructional process. More recently, this has been enhanced by evaluating the human factors issues involved in learning such as screen presentation (Duchastel, 1988). 
The final step is to integrate the systems development methodologies with those for human factors and intelligent CAL to produce an integrated system catering for the individual skills and requirements of the user (Dede, 1987; Carr, 1988).

\section{An Integrated Model}

A starting point for this approach is provided by Martin (1986b), who describes an "adaptive" model for user understanding which includes both human factors and training. This adaptive model is designed to pose questions to ascertain user knowledge levels and generate alternate processing paths to optimise performance (Martin, 1986b), and implies a system adapting to user needs rather than a user adapting the system to satisfy those needs (Edmonds, 1981). Although this approach emphasises the user-interface, it also includes the elementary components for an intelligent training system. However, the important considerations for adaptive systems are whether the additional design effort (as identified by Mantei and Teorey, 1988) and the apparent speed of novice learning justify the development of a user-oriented system. Although Martin (1986b, p29) concludes that the "design of business computer systems must gravitate towards the user", which supports the role of technology as a tool, the development effort required for such "people-oriented" systems cannot be ignored.

Moreover, it should be noted that the adaptive model proposed by Martin (1986b) does not include training as a component of the software application. Instead, training is portrayed in its traditional environmentseparate from the technology for which the training is designed. Given the developments in embedded CBT and intelligent CAL, the stage is set for software development based on the converging technologies of systems development, human factors and training.

A modification to the adaptive model is shown in Figure 2, with an iterative development process generating a software application which caters for data processing, user requirements and training to provide operational efficiency for the user. The implications of this model are that user understanding will be the major outcome, producing minimal errors in operation, increasing opportunity costs by minimising off-the-job training and achieving organisational objectives. This understanding will be achieved only if the complete system is continually evaluated and maintained.

An example of the user-interface provided by this model is shown in Figure 1, which illustrates the use of a context-sensitive ICAL which, when the user has made successive (abortive) attempts at a particular function, generates on-screen remedial information, provides the opportunity for natural language conversation and produces feedback when required. 


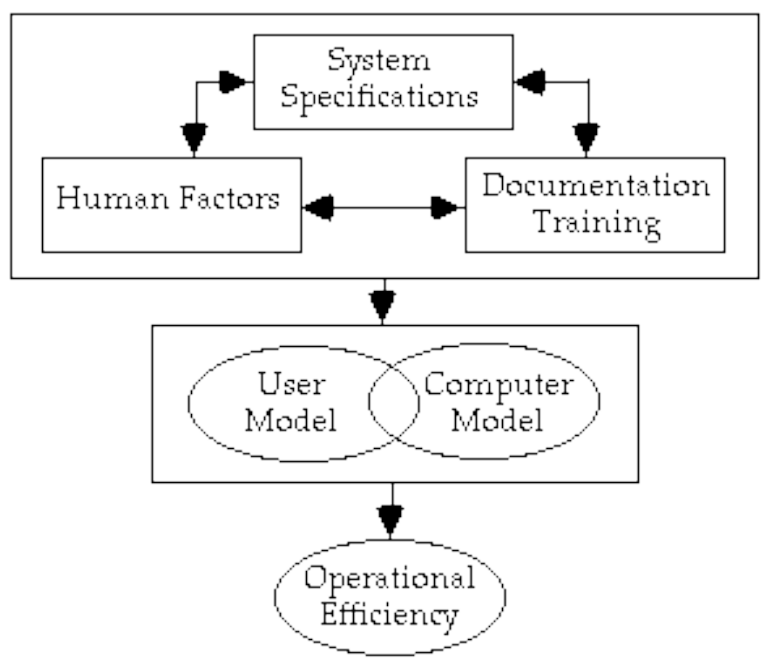

Figure 2: Integrating Systems Development, Human Factors and ICAL

However, it is the actual attributes of the model which demonstrate the potential for this form of software application, as described fully in Sims (in press). However, while the advantages of an integrated "peopleoriented" system will be seen through an application which provides an interface designed for user operation and education, considerable challenges remain.

Serious obstacles to overcome include the difficulties involved in extending the leading edge of research in artificial intelligence, and uncertainty about how much resources and time will be needed to develop products. Limits on the sophistication of user interfaces, on the scope of subject domains, and on current understanding are seriously constraining the effectiveness of current intelligent tutors, coaches, and empowering environments. (Dede, 1987, p181)

While these factors must be taken into account, the positive aspects of this integrated approach to systems and application software development will be operational efficiency achieved through users being prompted for incorrect actions, presented with option selections dependent upon their particular skill level and provided with remedial or orientation training when required.

This "people-oriented" model illustrates the advantages available to developers of computer systems through interacting with instructional 
and human factors engineers. While the requirements for storing user-data and monitoring user-performance may currently be reserved for mainframe environments, the continued developments in storage and retrieval technology will ensure the availability of this mode of processing.

\section{The Learner-Computer Interface II: Towards Context Based Courseware}

\section{Variables of Courseware Development}

An analysis of the research and development literature identified at least six variables which have affected the development of an effective learnercomputer interface. The omission of any one of these elements in courseware development will have a significant impact on the learning outcomes. However, these variables also provide a framework for refining the CAL instructional paradigm, with the expectation that CAL applications will increase in effectiveness as more is understood of the learner-computer interactions.

As all these variables are important in the courseware development process, it is suggested that applying each variable to the courseware structure will add to its effectiveness. The following analysis expands on these variables, providing a basis for evaluating the significance of each factor in the development and delivery of effective CAL systems. In addition, the importance of the variables in combination are considered as vital for the future success of CAL.

\section{Instructional Design}

There is increasing evidence that applying a systematic instructional methodology to the design and development of CAL will result in courseware acceptable to the targeted student population (Sims and Grant, 1987, Sims 1988b). Therefore, where CAL can be justified on cost or educational grounds it should be implemented according to a methodology which recognises foremost the instructional aspects of the resource, especially assessment, as this provides the learner with performance feedback. In fact, using a design methodology including assessment features will provide the basis for an effective learning resource, whatever the medium (Briggs, 1977; Romiszowski, 1984).

Traditionally, CAL has been developed according to instructional strategies such as Drill and Practice or Tutorial (Alessi and Trollip, 1985). However, there has been little development of models which are specific to the CAL medium that is, interactive, individualised instruction delivered on a computer-controlled monitor. The basic question is whether 
a new instructional model can be defined which will not only provide a valid instructional paradigm, but also take advantage of the technology in terms of learner-oriented displays and interactions. Support for this approach is seen in proprietary courseware developed by the writer (Sims, $1988 \mathrm{~b})$ which has proved effective by incorporating a validated instructional design (Romiszowski, 1984) and combining the five modes of courseware-tutorial, drill, test, game and simulation (Alessi and Trollip, 1985). This preliminary evidence suggests that an interactive model will provide an environment in which students feel comfortable with the learning resources.

As a learning resource, CAL must be designed not to be compared with alternate media, but to provide an environment in which learning is facilitated. Instructional design is one variable that will achieve this objective; however, it must be considered in relation to other developmental variables, such as technology, which provides the very foundation for educational computing.

\section{Technology}

Software and hardware technologies continue their rapid development, and CAL publications have emphasised the potential of "intelligent" learning applications which cater for the individual knowledge level and skill of the learner (Kearsley, 1987). An alternate software product which also provides a learner-centred option is "Hypercard", which gives the user or learner the browsing capability available with books in addition to the flexibility of the dynamic computer display. In this environment the student has the ability to search by interest or intuition, whereas the traditional CAL format more often than not guides the student through a pre-set instructional strategy. As the majority of CAL packages conform to traditional instructional paradigms (Drill and Practice, Tutorial etc.) and have not demonstrated new approaches in the presentation of information/instructional content, it is important that technological developments which can assist the learning process are incorporated into the instructional model.

For example, advances in memory and storage facilities have resulted in Computer-Based Training (CBT) applications implementing Embedded CBT, which is used by organisations to provide advanced on-line help and training facilities, with the user able to request training on a "live" system when required (Kearsley, 1985; Bentley, 1988). Additionally, developments in Artificial Intelligence and Expert Systems have seen considerable changes in CAL resources, with instruction based on individual requirements and more efficient communication (Kearsley, 1987). Computer technology has also accelerated the access to information through videodisc and CD-ROM, providing the opportunity to further enhance the learning environment. 
With the expansion in technology to almost all business and educational environments, there is a growing demand for computer systems which will adapt to individual skill levels. Developers of courseware must assess the benefits of technology in terms of learner requirements, and integrate them with the courseware where appropriate to learner demands.

\section{Cognitive Styles}

In comparison with other elements of CAL development, the individual learning preferences of students received little attention. However, there is evidence to suggest that individuals with particular learning styles will prefer specific CAL presentation formats. For example, Martin (1982) found that field-dependent subjects were more likely to benefit from explanatory feedback, while Burger (1985) reported that there was no correlation between learning-style and CAL preference. The latter study however failed to indicate whether the lesson itself was suitable for the particular learning styles being evaluated. Nevertheless, the results suggest that CAL may either be of benefit only to those with particular learning styles, or that specific courseware formats are required to match variations in an individual's cognitive style.

The importance of this variable is in providing inputs for the design of the learner-interface, such that the courseware will adapt to individual processes. This need not demand the development of different versions of the content (textual vs. graphical), but the use of technology to process the content data such that students may have the option to view the display under different conditions.

With the current emphasis on cognitive psychology and the user interface (Gardiner and Christie, 1986), it is apparent that the importance of the individual learner in the CAL environment will only increase in significance.

\section{Screen Design}

The CAL field is now some 30 years old, and there are still no widely accepted standards for presentation of text and graphics. While certain "cookbook" publications provide basic guidelines and/or standards for screen design, they tend to emphasise the text component rather than the use of a dynamic instructional medium (Isaacs, 1987; Rambally and Rambally, 1987, Gillingham, 1988). With the implicit assumption that a single display format will not affect the learner-machine interactions, the major omission from these works is an allowance for individual preferences, as emphasised by the following remarks:

Computers in the future are likely to become a preferred means for the student to find information, and it seems important to start considering the display features of this medium in terms of how the student interacts with it in order to learn. (Duchastel, 1988, p58) 
The screen design of CAL resources is a major determinant of effective communication; however, the developer of CAL has the option to vary this display to cater for individual differences. The extent to which this variation affects learning, retention and transfer of skills however is yet to be established.

\section{Transfer and Retention}

Of perhaps most importance educationally is the actual learning which results from the use of CAL resources, in terms of effective transfer of training (Clark and Voogel, 1985) as well as the facilitation of recall and the quality of retention (Wood et al, 1987; Umanath and Scamell, 1988).

In the majority of research articles, CAL has been examined on a single condition rather than in terms of practical application or through longitudinal considerations. In other words, the transfer of training and/or retention of content through CAL resources has not been sufficiently evaluated. As it is presumed that the purpose of CAL is to assist student learning, it is equally important that its ability to enforce retention is established.

One reason for this may be a lack of assessment items within the courseware. Although CAL typically adopts a mastery approach, the writer has personally been a student in a complete CAL curriculum which could be mastered, not by learning, but by noting the answers to questions, and applying them on subsequent tests! The quality of CAL presentation must be matched with its effect on real learning. The implication for courseware development is that evaluation needs to extend beyond an immediate post-test to a consideration of individual students in terms of their ability to generalise and transfer the skills learnt.

\section{The Learner-Computer Interface}

In identifying and accepting these variables, it is apparent that CAL is moving from technology-significant and instructor-oriented media towards the learner. The effect of this is increased importance being placed on the quality of the interface with the technology. One such area where this has received attention is in the ability of the resource to adapt to individual requirements, thereby maximising the effectiveness of communication and interaction between the learner and the technology.

For example, one of the popular techniques to enhance the learnercomputer interface is the menu, which provides the student with a selection of choices for study. However, the menu does not provide the student with an easy reference to their "position" in the course, as provided by the "map" in the demonstration versions of Hypercard. To assess the benefits of enhanced learner reference points, development work currently being completed by the writer involves implementing a 
course "map" into courseware. This has evolved from a colour-coded map to icon-based representations to act as a visual cue and maximise the transparency of the interface.

\section{Design Criteria}

Developments in the learner-computer interface will result from an application of each of the variables identified to the development of courseware which is not only instructionally effective, but also provides the student with a representation of completion status as well as tasks to be completed.

The identification of these variables provides a framework for an analysis of CAL in terms of instructional theory and the processing capabilities of the individual. From the brief analysis it would appear that no single factor contributes to the quality and effectiveness of CAL, giving rise to the following model, illustrated in Figure 3, which describes CAL effectiveness as a function each variable:

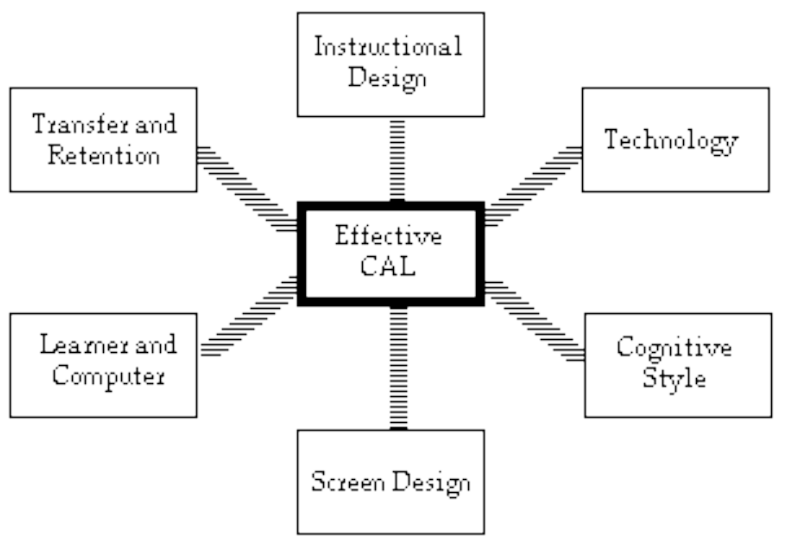

Figure 3: Integrated CAL Development Model

\section{Conclusion}

From the above discussion, two major opportunities are evident for CBT. First it is apparent that information systems and instructional systems are converging on a track which will generate "people oriented" systems catering for varying user skills, the range of interactions required between user and system and the knowledge level of the user. While the problems in developing such systems will be based on current technological capabilities and the motivation of organisations to provide an efficient, operational system, the ever-increasing literature on human factors, cognitive psychology and the user-interface emphasises the importance of this development. 
Second, the application of specific variables to the development of courseware will result in the introduction of an "interactive" model for CAL with presentation based on a transparent learner-computer interface whereby the learner works with the content, with minimal interference from the sequence controls.

The implications are that CAL is rapidly moving to a learner orientation, and the projection of this development is CAL where the learner is actively involved in the learning sequence.

\section{References}

Alessi, S. M. and Trollip, S. R. (1985). Computer-Based Instruction: Methods and Development. New Jersey: Prentice-Hall.

Bentley, T. J. (1987). ECBT. Banking and Financial Training, 15-16.

Bentley, T. J. (1988). A Guide to Embedded Computer Based Training. Training and Development in Australia, 15(1), 19-22.

Black, W. J. (1986). Intelligent Knowledge Based Systems. London: Van Nostrand Reinhold.

Briggs, L. J. (Ed) (1977). Instructional Design. Englewood Cliffs: Educational Technology Publications.

Burger, K. (1985). Computer-Assisted Instruction: Learning Style and Academic Achievement. Journal of Computer-Based Instruction, 12(1), 2122.

Carr, C. (1988). Skilling America: The Potential of Intelligent Job Aids. Educational Technology, 28(4), 22-25.

Clark, R. E. and Voogel, A. (1985). Transfer of Training Principles for Instructional Design. Educational Communication and Technology, 33(2), 113-123.

Davis, G. B. and Olson, M. H. (1985). Management Information Systems. New York: McGraw-Hill.

Dede, C. (1987). Artificial Intelligence Applications to High Technology Training. Educational Communication and Technology, 35(3), 163-181.

Duchastel, P. C. (1988). Display and interaction features of instructional texts and computers. British Journal of Educational Technology, 19(1), 58-65.

Edmonds, E.A. (1981). Adaptive Man-Computer Interfaces. In Coombs, M. J. and Alty, J. L. (Eds), Computing Skills and the User Interface. London: Academic Press.

Gardiner, M. M. and Christie, B. (Eds) (1987). Applying Cognitive Psychology to User-interface design. Chichester: Wiley.

Gillingham, M. G. (1988). Text in Computer-Based Instruction: What the Research Says. Journal of Computer-Based Instruction, 15(1), 1-6.

Isaacs, G. (1987). Text Screen Design for Computer-Assisted Learning. British Journal of Educational Technology, 18(1), 41-51.

Kearsley, G. (1985). Embedded Training: The New Look of ComputerBased Instruction. Machine-Mediated Learning, 1(3), 279-296. 
Kearsley, G. P. (Ed) (1987). Artificial Intelligence and Instruction. Reading, Massachusetts: Addison-Wesley.

Mantei, M. M. and Teorey, T. J. (1988). Cost/Benefit Analysis for Incorporating Human Factors in the Software Lifecycle. Communications of the ACM, 31(4), 428-439.

Martin, M. (1982). Cognitive Styles and Instructional Variables. Presented to the 1982 Annual Meeting of the American Educational Research Association, New York.

Martin, M. P. (1986a). The Human Connection in Systems Design: Part IIIScreen Design. Journal of Systems Management, 37(10), 15-22.

Martin, M. P. (1986b). The Human Connection in Systems Design: Part IVAdaptive Models. Journal of Systems Management, 37(10), 23-29.

Rambally, G. K. and Rambally, R. S. (1987). Human Factors in CAI Design. Computers and Education, 11(2), 149-153.

Romiszowski, A. J. (1984). Producing Instructional Systems. London: Kogan Page.

Sawyer, T. A. (1985). Human Factors Considerations in ComputerAssisted Instruction. Journal of Computer-Based Instruction, 12(1), 17-20.

Sims, R. (1986). On the Production of High-Quality Courseware. In G. Bishop and W. van Lindt (Eds), CALITE 86 Proceedings. University of Adelaide.

Sims, R. (1988a). Futures for Intelligent Computer-Assisted Learning. Proceedings of the 1988 Australian Computer Education Conference, Perth, Australia.

Sims, R. (1988b). Commercial CBT Development: A Case Study. CBT Training Workshop. Australian Computer Society (NSW Branch).

Sims, R. (in press). Bringing it all Together: Perspectives on PeopleOriented Information Systems. 22nd Hawaii International Conference on Systems Sciences. Hawaii, January 1989.

Sims. R. and Grant, P. (1987). Instructional Design: The Hidden Factor. In J. Barrett and J. Hedberg (Eds), Intelligent Computer-Assisted Learning. CALITE 87 Proceedings, Sydney.

Umanath, N. S. and Scamell, R. W. (1988). An Experimental Evaluation of the Impact of Data Display Format on Recall Performance. Communications of the ACM, 31(5), 562-570.

Wood, E., Pressley, M., Turnure, J. E., and Walton, R. (1987). Enriching Children's Recall of Picture-Dictionary Definitions With Interrogation and Elaborated Pictures. Educational Communication and Technology, 35(1), 43-52.

Please cite as: Sims, R. (1988). Futures for computer-based training: Developing the learner-computer interface. Australian Journal of Educational Technology, 4(2), 123-136. http:/ / www.ascilite.org.au/ajet/ajet4/ sims.html 